\title{
A step-by-step guide to transseptal valve-in-valve transcatheter mitral valve replacement
}

\author{
Morgan T. Harloff ${ }^{1}$, Muntasir Chowdhury ${ }^{1}$, Sameer A. Hirji ${ }^{1}$, Edward D. Percy ${ }^{1}$, Farhang Yazdchi $^{1}$, \\ Hunbo Shim ${ }^{1}$, Alexandra A. Malarczyk ${ }^{1}$, Piotr S. Sobieszczyk ${ }^{2}$, Ashraf A. Sabe ${ }^{1}$, Pinak B. Shah ${ }^{2}$, \\ Tsuyoshi Kaneko ${ }^{1}$
}

${ }^{1}$ Division of Cardiac Surgery, Brigham and Women's Hospital, Harvard Medical School, Boston, MA, USA; ${ }^{2}$ Division of Cardiovascular Medicine, Brigham and Women's Hospital, Boston, MA, USA

Correspondence to: Tsuyoshi Kaneko. Division of Cardiac Surgery, Brigham and Women's Hospital, 15 Francis Street, Boston, MA 02115, USA.

Email: tkaneko2@partners.org.

\begin{abstract}
With the recent success of transcatheter aortic valve replacement (TAVR), transcatheter options for the management of mitral valve pathology have also gained considerable attention. Valve-in-valve (ViV) transcatheter mitral valve replacement (TMVR) is one such technique that has emerged as a safe and effective therapeutic option for patients with degenerated mitral valve bioprostheses at high-risk for repeat surgical mitral valve replacement. Several access strategies, including trans-apical, transseptal, trans-jugular, and trans-atrial access have been described for ViV-TMVR. Initial experiences were performed primarily via a trans-apical approach through a left mini-thoracotomy because it offers direct access and coaxial device alignment. With the advancements in TMVR technology, such as the development of smaller delivery catheters with high flexure capabilities, the transseptal approach via the femoral vein has emerged as the preferred option. This technique offers the advantages of a totally percutaneous approach, avoids the need to enter the thoracic cavity or pericardial space, and provides superior outcomes compared to a trans-apical approach. In this review, we outline key aspects of patient selection, imaging, procedural techniques, and examine contemporary clinical outcomes of transseptal ViV-TMVR.
\end{abstract}

Keywords: Valve-in-valve (ViV); transcatheter mitral valve replacement (TMVR); transseptal

Submitted Jun 01, 2020. Accepted for publication Dec 22, 2020.

doi: 10.21037/acs-2020-mv-104

View this article at: http://dx.doi.org/10.21037/acs-2020-mv-104

\section{Introduction}

Surgical mitral valve replacement remains the standard of care for patients with severe mitral valve disease when mitral valve repair is not feasible (1). Subsequent degeneration of the bioprosthetic valve however, can limit long-term success (1). When structural valve degeneration occurs, some patients may ultimately require repeat surgical mitral valve replacement, a high-risk operation associated with high mortality and major complications (2). As a result, interest in the development of innovative and less invasive approaches to treat bioprosthetic mitral valve deterioration is growing.

The success of transcatheter aortic valve replacement
(TAVR) over the last decade has spurred interest in the production of similar percutaneous options for the management of severe mitral valve disease. Valve-invalve ( ViV) TMVR is one such therapeutic option that has emerged as a safe and reproducible alternative for patients with a degenerated bioprosthesis at high-risk for repeat surgical mitral valve replacement (3). The ring of a degenerated bioprosthesis serves as a reliable anchoring point for a transcatheter valve, thus avoiding one of the major limitations associated with TMVR in the native mitral annulus (3). Early experiences with ViV-TMVR were conducted via a trans-apical approach through a left mini-thoracotomy as it offers direct access and device 


\begin{tabular}{ll} 
Table 1 Contraindications for transseptal ViV-TMVR \\
\hline Absolute contraindications & Relative contraindications \\
\hline - Infective endocarditis & - Narrow LVOT \\
- Dehiscence of the bioprosthesis & - Bioprosthetic PVLs \\
- Bioprosthetic thrombosis & - Thrombus within the right or left atrial cavity \\
- Thrombus at the intra-atrial septum & - Severe patient-prosthesis mismatch \\
- Interrupted IVC & - Prior atrial septal defect repair \\
& - Prior mitral valve repair via a transseptal approach \\
\hline $\begin{array}{l}\text { Source: references (8,9). ViV, valve-in-valve; TMVR, transcatheter mitral valve replacement; LVOT, left ventricular outflow tract; PVL, } \\
\text { paravalvular leak; IVC, inferior vena cava. }\end{array}$
\end{tabular}

coaxiality (4). However improved technology in smaller and steerable delivery systems, has allowed for the development of transseptal ViV-TMVR. This fully percutaneous approach via the femoral vein avoids the mini-thoracotomy and the need to enter the thoracic cavity or pericardial space. Early experience with transseptal ViV-TMVR has shown that it is not only safe and effective, but also offers the advantage of less morbidity and recovery time compared to conventional surgery and trans-apical TMVR $(5,6)$.

Herein, we discuss important aspects of transseptal ViVTMVR with an Edwards SAPIEN 3 Transcatheter Heart Valve System (Edwards LifeSciences Corp., Irvine, CA, USA) within the framework of the following sequential steps: (I) patient selection; (II) imaging; (III) preparation; (IV) vascular access; (V) transseptal puncture; (VI) valve deployment; (VII) closure.

\section{Procedural technique}

\section{Patient selection}

Patient selection is critical to conducting successful transseptal ViV-TMVR. The heart team should consider this approach for patients with a degenerated mitral valve bioprosthesis at high-risk for repeat open-heart surgery. However, several factors may prevent some patients from being optimal candidates. For instance, if the patient has had a previous atrial septal defect repair or mitral valve surgery via a transseptal approach, caution must be taken and the transseptal approach may not be feasible. Those with a small left ventricular outflow tract (LVOT) and a long, calcified anterior mitral valve leaflet may not be good candidates either as they are at increased risk for LVOT obstruction, the most feared and potentially life-threatening complication associated with TMVR (7). Additionally, patients with severe patient-prosthesis mismatch should be carefully selected as placing a transcatheter valve inside an already small bioprosthesis can exacerbate the problem (8). The presence of endocarditis, severe paravalvular leak (PVL), thrombosis or dehiscence of the bioprosthesis may also contraindicate the procedure $(8,9)$. The relative and absolute contraindications for transseptal ViV-TMVR are summarized in Table $1(8,9)$.

\section{Imaging}

Pre-operative computed tomography (CT) TMVR is mandatory to assess the potential risk for iatrogenic LVOT obstruction. Simulation with a virtual transcatheter valve in the mitral annulus on CT can predict the hypothetical LVOT area (neo-LVOT) after ViV-TMVR (7). Projected neo-LVOT area $\leq 1.7 \mathrm{~cm}^{2}$ carries high sensitivity and specificity for postprocedural LVOT obstruction (7). As such, strategies to reduce the risk of LVOT obstruction may need to be considered to minimize this hazard. CT can also be used to measure the internal diameter of the old bioprosthesis as a reference size for the transcatheter valve to be inserted (10). Although rare, CT can also identify an interrupted inferior vena cava (IVC), a phenomenon that would render transseptal ViV-TMVR infeasible. Furthermore, transesophageal echocardiography (TEE) should be used to identify the mechanism for bioprosthetic failure and assess the anatomy of the interatrial septum. If the septum cannot be identified on TEE, transseptal ViVTMVR is not feasible. TEE can also be used to increase 


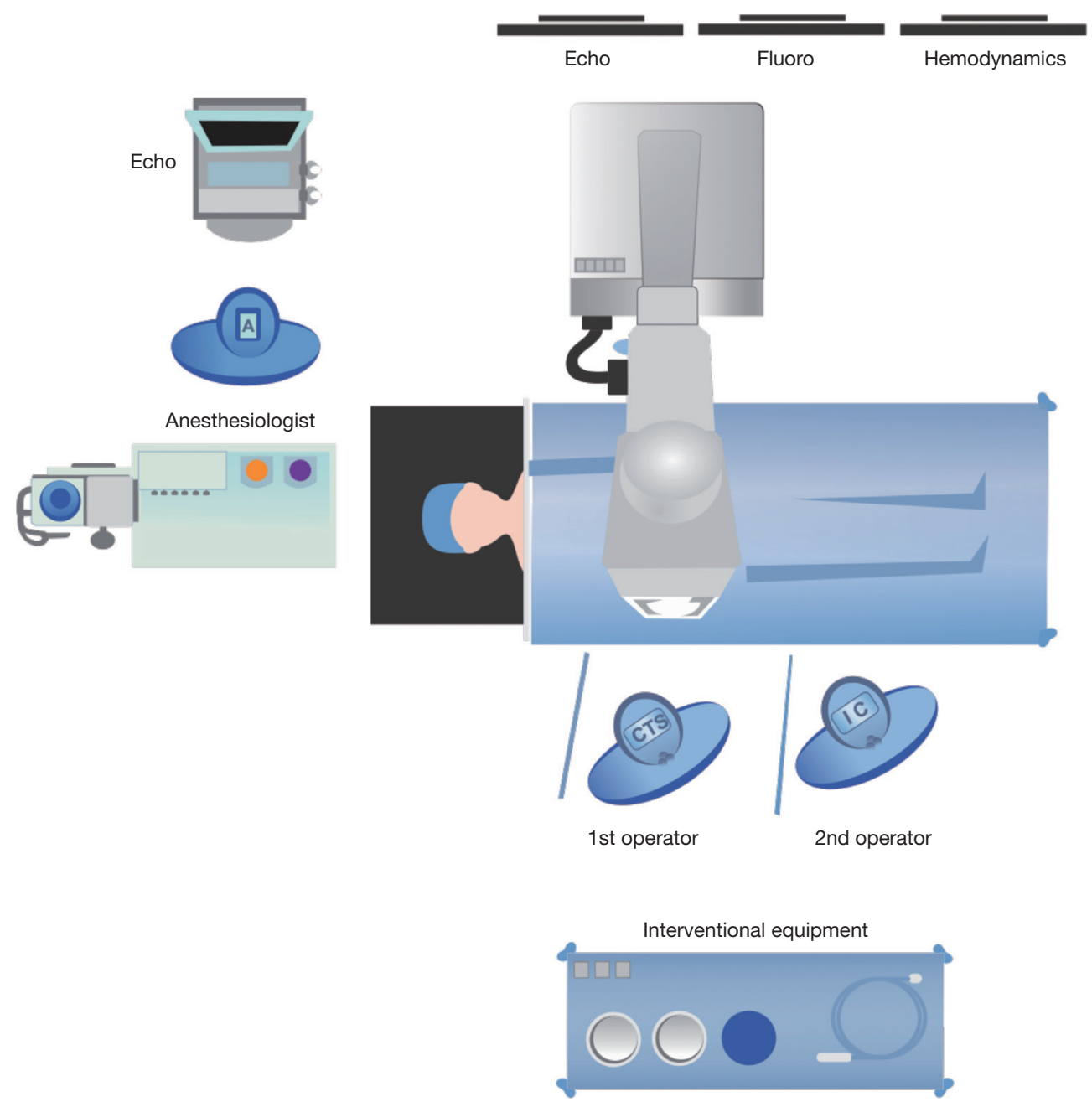

Figure 1 Room set-up depicting both providers standing on the patient's right side with the fluoroscopic monitors on the patient's left.

the safety and success rate in patients with a thickened or hypertrophic atrial septum (9). Coronary angiogram should also be performed in patients at high-risk for LVOT obstruction to identify the first septal perforator in the event that pre-emptive alcohol septal ablation needs to be performed (11).

\section{Preparation}

The patient is brought to the catheterization laboratory or hybrid fluoroscopy operating room and placed in the supine position. The proposed position of the room is shown in Figure 1. A radial arterial line is placed with a 20 -gauge catheter for continuous hemodynamic monitoring during the procedure. After induction, a TEE probe is advanced into the esophagus for pre- and post-procedural evaluation of the mitral valve. Both groins are prepped, and the patient is draped per standard surgical convention.

\section{Vascular access}

The bilateral common femoral veins are accessed with a 6 French (Fr) sheath under ultrasound (US) guidance via the Seldinger technique. Then, a Perclose Proglide (Abbott Laboratories, Chicago, IL, USA) device is preemptively placed in the right femoral vein for eventual suturemediated closure of the venotomy that will be used for insertion of the large-bore sheath. Next, a steerable vascular access sheath such as the Agilis NxT Steerable Introducer (Abbott Laboratories, Lake Bluff, IL, USA) is advanced into 


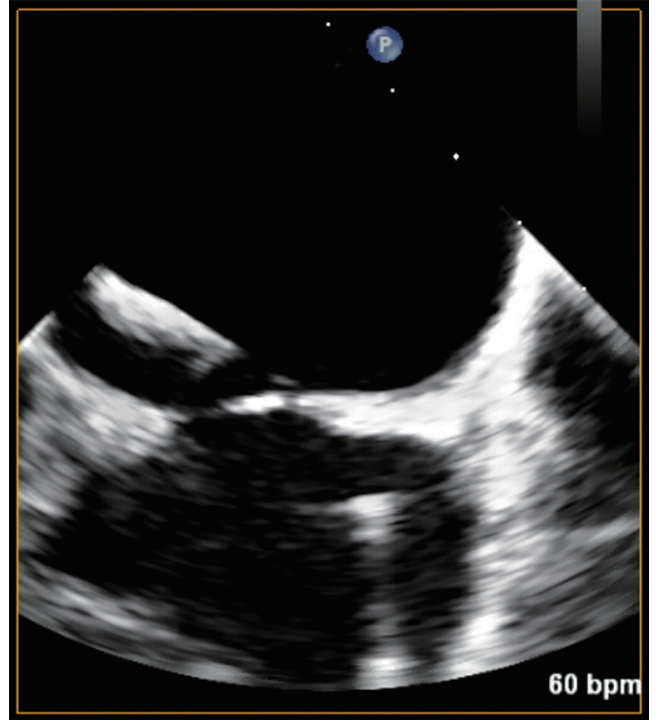

Figure 2 Intra-op TEE showing the BRK Transseptal needle approaching the interatrial septum. TEE, transesophageal echocardiography.

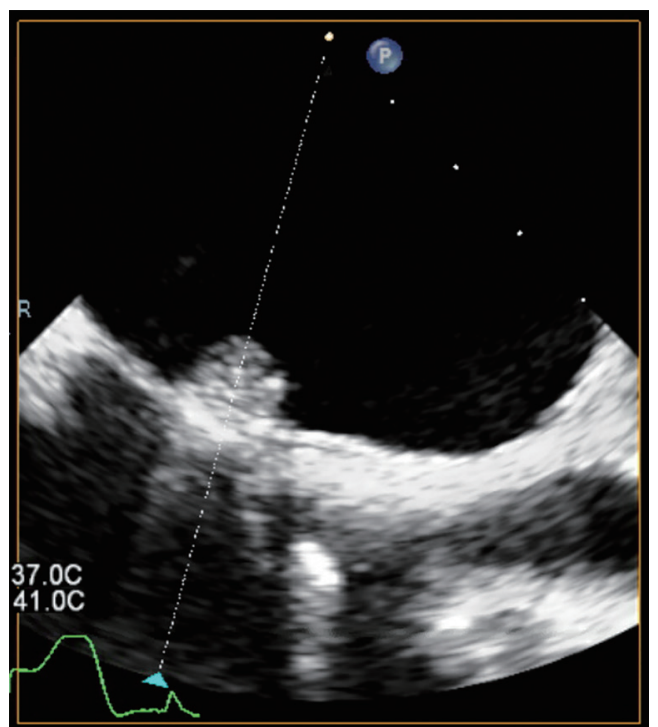

Figure 3 Intra-op TEE showing the BRK Transseptal needle piercing the interatrial septum. TEE, transesophageal echocardiography.

the right femoral vein. Under fluoroscopy, the Agilis NxT Steerable Introducer (Abbott Laboratories, Lake Bluff, IL, USA), is floated up the IVC and positioned at the junction of the IVC and right atrium (RA).

\section{Transseptal puncture}

A dose of 5,000 units of heparin is given systemically, then, a Mullins sheath (Cook Group, Bloomington, IN, USA) is inserted into the right femoral vein and advanced into the RA. An NRG transseptal needle (Baylis Medical, Burlington, MA, USA) is advanced through this sheath and gently directed toward the fossa ovalis under TEE and fluoroscopic guidance (Figure 2). The atrial septum is punctured with radiofrequency transmitted through the transseptal needle and the Agilis sheath is advanced into the left atrium (LA) (Figure 3). Before proceeding further, the introduction of the sheath into the LA should be confirmed by transduction of LA pressures on the monitor. The transseptal needle is withdrawn and an Inoue wire (Toray Medical Co., Ltd., Tokyo, Japan) is advanced through the sheath into the LA. With careful attention to keep the wires in the LA, the Agilis sheath is advanced further into the LA. Next, the patient is systemically heparinized. After confirming therapeutic activated clotting time (ACT) with a goal of 250-350 seconds, the Agilis sheath is steered towards the left ventricular apex and a standard J-wire is passed towards the left ventricle (LV). If there is a concern for chordal entanglement with the J-wire, a pulmonary artery catheter can be advanced across the wire with the balloon inflated to confirm the lack of entanglement. Then, a pigtail catheter is advanced into the $\mathrm{LV}$ over the J-wire. Finally, a temporary transvenous pacemaker is inserted through the left femoral venous sheath, floated up to the heart, and anchored into the trabeculae of the right ventricle.

\section{Valve deployment}

With standard wire exchange techniques, a Safari wire (Boston Scientific Corp., Marlborough, MA, USA) is advanced through the pigtail catheter, across the mitral valve, and into the apex of the LV. Over the Safari wire, a 14 Fr Edwards eSheath (Edwards LifeSciences Corp., Irvine, CA, USA) is inserted. The interatrial septum is then dilated with a $14 \mathrm{~mm}$ balloon (Figure 4). The balloon is withdrawn and an Edwards SAPIEN 3 Transcatheter Heart Valve System (Edwards LifeSciences Corp., Irvine, CA, USA) is advanced into the IVC. It is important to mount the valve on the delivery catheter with the skirt towards the handle, opposite the direction in which it would be loaded for TAVR. To load the valve, the sheath typically needs to be moved back to give enough room. After loading the valve on the balloon, the entire sheath is rotated 180 degrees 


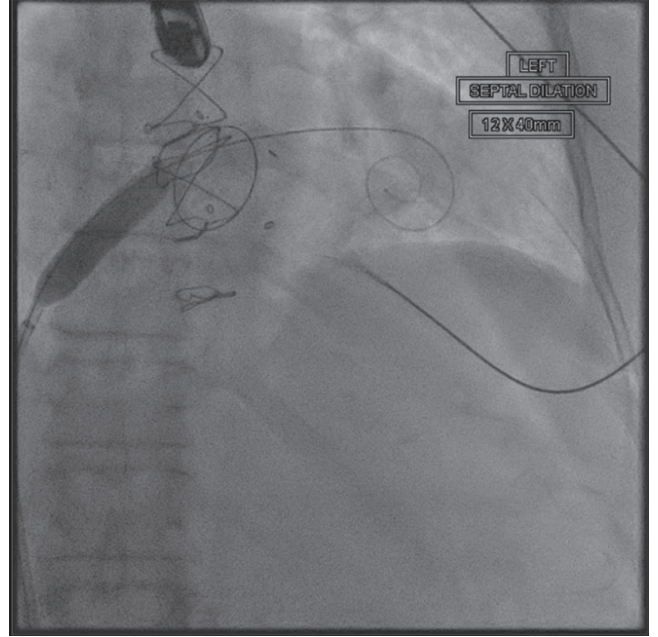

Figure 4 Intra-op cath showing balloon dilation of the interatrial septum with a $14 \mathrm{~mm}$ balloon over the Safari wire that has been advanced through interatrial septum, across the bioprosthetic mitral valve, and into the left ventricular apex.

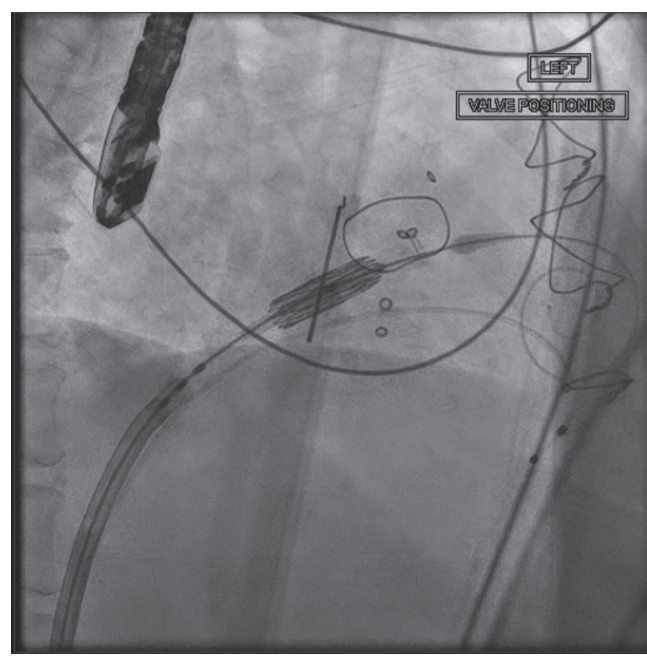

Figure 5 Intra-op cath showing positioning of the transcatheter valve within the ring of the old bioprosthetic mitral valve.

clockwise. This maneuver allows the flexing mechanism of the device to be angled rightwards, opposite of the leftward curve in TAVR. Then, the device is advanced across the atrial septum, and into the LA. Additional curve may be needed to advance the valve into the mitral valve (Figure 5).

After satisfactory positioning, the valve is deployed under rapid ventricular pacing (Figure 6). The valve typically is not perfectly coaxial to the annular plane but autocorrects during valve inflation. Therefore, the inflation must be slow and the person closest to the valve must be prepared to adjust the position during valve inflation. Following valve deployment, TEE is used to assess valve position, motion of the leaflets, trans-mitral gradients, presence of PVLs, and gradient across the LVOT (Figure 7). If PVL is present, the valve can be further dilated by injecting additional contrast into the delivery system under fluoroscopy. Completion TEE is used to reassess PVL after post balloon dilatation.

\section{Closure}

The iatrogenic septal defect is not routinely closed. However, in the presence of a significant right-to-left inter-atrial shunt or pulmonary hypertension with right ventricular failure, the septostomy can be closed with a percutaneous closure device. Next, the temporary pacing wire, delivery system, and sheaths are withdrawn from the femoral veins. Protamine is instituted and the Perclose Proglide device in the right femoral vein is tied down. Manual compression is applied over the left venous access site for hemostasis. We typically place a subcutaneous $\mathrm{u}$-stitch to secure hemostasis. Finally, the radial artery sheath is removed and a TR band (Terumo Interventional Systems, Somerset, NJ, USA) is applied over the wrist to obtain hemostasis at the radial artery access site.

\section{Comments}

\section{Clinical results}

Significant progress has been made over the last decade with the transcatheter treatment of valvular heart disease. TAVR is now an established therapeutic option for patients with severe symptomatic aortic stenosis in all surgical risk profiles $(12,13)$. Although ViV-TMVR is at a much earlier phase of development, it is a rapidly evolving technology that offers an alternative for patients with bioprosthetic mitral valve degeneration at high-risk for conventional repeat mitral valve surgery. In fact, recent studies have demonstrated safety and efficacy in such patients with deteriorated mitral bioprostheses who are poor candidates for repeat surgery $(3,14)$. Given these results, the Food and Drug Administration (FDA) approved ViV-TMVR for a deteriorated mitral valve prosthesis in high-risk patients in 2017.

During its nascent stages, ViV-TMVR access was limited by the high-profile delivery systems that were needed to accommodate a large transcatheter mitral valve 

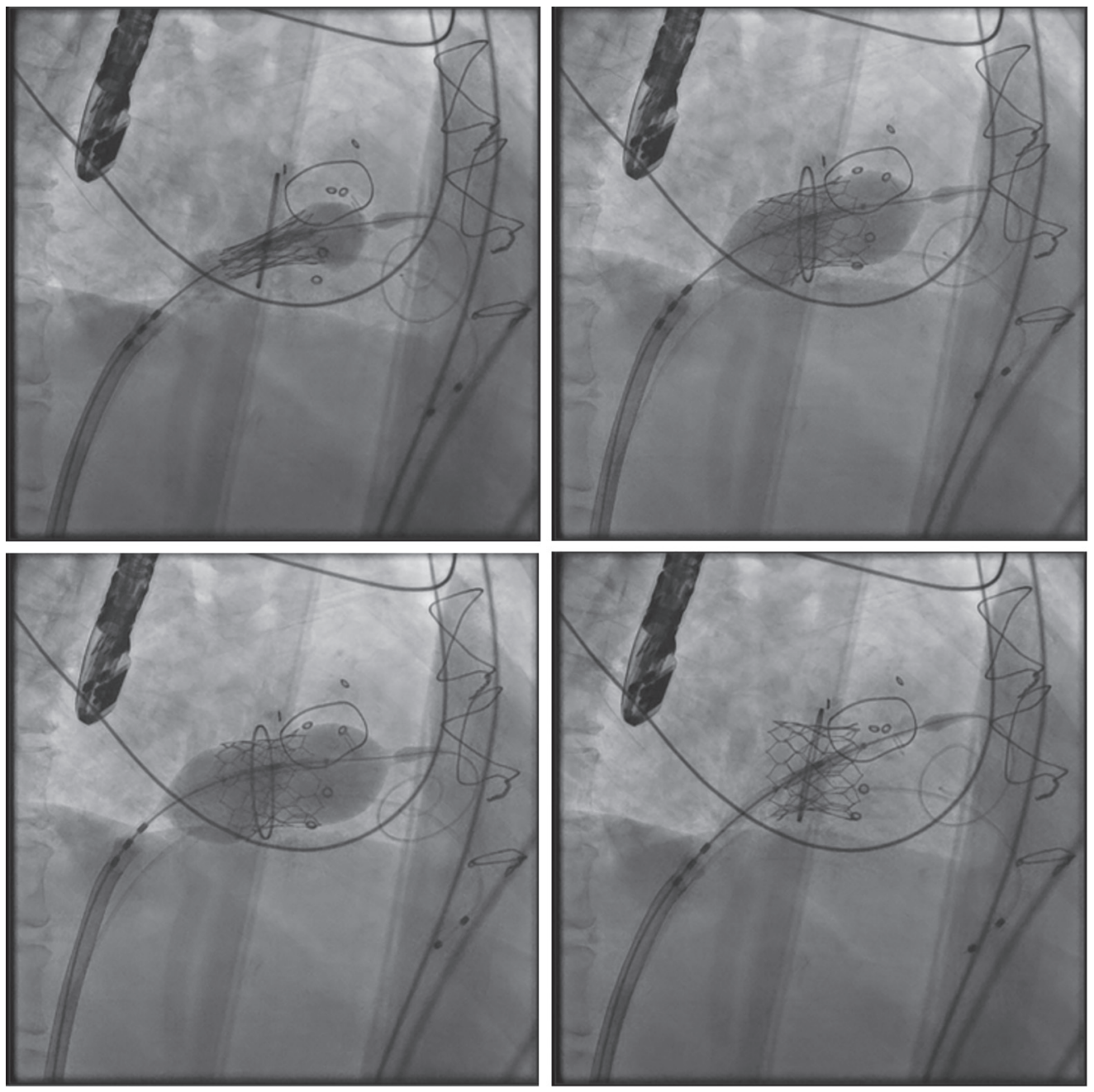

Figure 6 Intra-op fluoroscopy showing gradual balloon inflation and deployment of the transcatheter valve inside the bioprosthetic mitral valve.

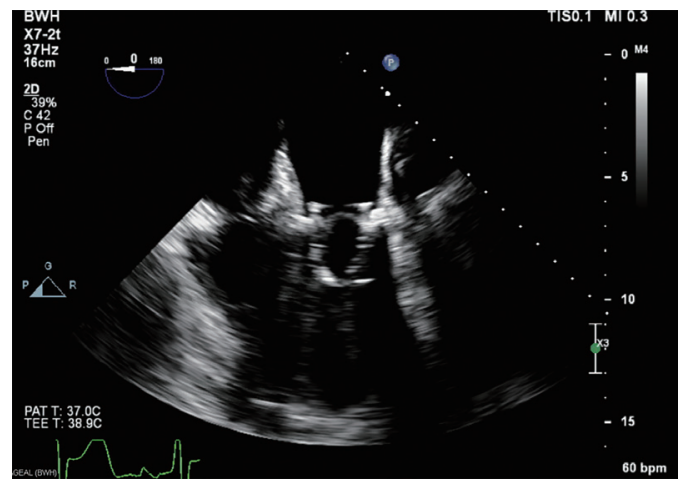

Figure 7 Intra-op transesophageal echocardiogram showing successful transcatheter valve deployment. prosthesis (4). Additional challenges included engineering a delivery system that could negotiate the extreme angle within the LA required to reach the native mitral valve from a transseptal approach. For these reasons, trans-apical access emerged as the initial access site of choice in the early phase of ViV-TMVR. Familiarity with trans-apical access from experience with TAVR through a mini-thoracotomy and the straight pathway to the mitral valve also factored into the early preference for trans-apical access. However, our vast experience with trans-apical access in TAVR has shown that this approach is associated with inferior outcomes compared to trans-femoral access (15). For example, transapical access has been associated with a higher degree of 
myocardial damage and harmful effects of the thoracotomy compared to trans-femoral access in the TAVR literature $(1,16)$. In addition, trans-apical access is associated with higher rates of pleural effusion, bleeding, atrial fibrillation and prolonged intubation (15-17).

Advancements in TMVR technology, including the development of steerable delivery catheters with high flexure capabilities, have led to the transition to a transseptal approach, a more desirable technique accomplished via percutaneous access of the femoral vein. According to Whisenant and colleagues who examined 1,576 transcatheter mitral ViV cases using the Sapien 3 TAVR valve (Edwards Lifesciences) captured from the Society of Thoracic Surgeons/American College of Cardiology TVT Registry between June 2015 and August 2019, the use of transseptal access increased over the study period and ultimately surpassed trans-apical access in the first quarter of 2016 (14). In fact, $84.1 \%$ of the 1,529 procedures overall were performed via transseptal access (14). There are a number of reasons for this phenomenon.

In direct head-to-head comparison with trans-apical access in the same study, Whisenant et al. found several key advantages in favor of transseptal access. Although transseptal was associated with higher fluoroscopy time compared to trans-apical access (37 vs. 18.2 minutes, $\mathrm{P} \leq 0.0001)$, trans-apical access required conversion to open surgery more frequently than transseptal $(2.5 \%$ vs. $0.7 \%$, $\mathrm{P}=0.03$ ) (14). Technical success was not statistically different between trans-apical and transseptal at $97.1 \%$ and $94.6 \%$, respectively (14). The transseptal group less frequently resulted in cardiovascular death at 30 -day $(2.1 \%$ vs. $5.2 \%$, $\mathrm{P}=0.01)(14)$. All-cause mortality at 1 -year also clearly favored the transseptal group over trans-apical access $(15.8 \%$ vs. $21.7 \%$; HR, 0.67; 95\% CI, 0.47-0.97) (14). Furthermore, data from the Valve-in-Valve International Data (VIVD) Registry showed an improvement in left ventricular ejection fraction (LVEF) after transseptal ViV-TMVR in patients with baseline LVEF $\leq 50 \%$ compared to trans-apical access (absolute average difference 5.5\%, $\mathrm{P}=0.03$ ) (18).

\section{Advantages and disadvantages}

Transseptal ViV-TMVR offers a safe and effective strategy for the treatment of a degenerated bioprosthesis in patients at high-risk for repeat surgical mitral valve replacement (19). It obviates the need for cardiopulmonary bypass and eliminates the need to excise a deteriorated bioprosthesis, potentially reducing the risk of permanent pacemaker placement or injury to the circumflex artery associated with reoperative MVR (2). Transseptal ViV-TMVR also offers a number of advantages over trans-apical access. First, it is more appealing to patients because it is less invasive, eliminates the need for a thoracotomy, and avoids the trauma to the LV (18). Second, the femoral vein is of large caliber and almost always allows for safe introduction of a large sheath (18). Third, access from the venous system allows low pressure closure and low risk of vascular injury.

The Achilles heel of ViV-TMVR in general, irrespective of access choice, is iatrogenic LVOT obstruction. ViVTMVR-induced LVOT obstruction, defined as an LVOT peak gradient increase of $\geq 10 \mathrm{mmHg}$ post-TMVR, occurs when the metal frame of the transcatheter valve pushes the anterior leaflet of the old bioprosthesis toward the interventricular septum, consequently narrowing the LVOT $(20,21)$. Untreated, hemodynamic instability results due to impediment of blood flow across the LVOT during systole (21). Some techniques have been described to overcome the risk of LVOT obstruction. For example, the intentional transcatheter laceration of the anterior mitral valve leaflet (LAMPOON) technique utilizes radiofrequency energy to lacerate the anterior mitral leaflet so that it cannot obstruct the LVOT after ViV-TMVR (22). Pre-emptive alcohol septal ablation has also been described to increase the area of the predicted neo-LVOT in patients that would otherwise be excluded from TMVR due to prohibitive risk of LVOT obstruction (11). Neither technique has yet been approved by the FDA however.

Compared to trans-apical access, achieving coaxial alignment with the mitral annulus is more challenging with the transseptal approach. As such, more adjustments may need to be made during balloon inflation to ensure proper coaxial alignment of the transcatheter valve. The durability of transcatheter valves in the mitral position also remain unknown. Thus, the long-term outcomes of ViV-TMVR warrant further study.

\section{Conclusions}

Advancements in TMVR technologies and preference for a less invasive approach have allowed the application of transseptal ViV-TMVR to grow rapidly among patients with a degenerated mitral bioprosthesis at high or prohibitive surgical risk. The outcomes reported have shown this to be 
a safe and feasible procedure, though further studies with long-term outcomes are needed.

\section{Acknowledgments}

Funding: None.

\section{Footnote}

Conflicts of Interest: Dr. TK, MD is a speaker for Edwards LifeSciences (Irvine, California), Abbott Laboratories (Lake Bluff, Illinois), and Medtronic (Minneapolis, Minnesota) and a consultant for Baylis (Burlington, Massachusetts) and 4C Medical (Maple Grove, Minnesota). The other authors have no conflicts of interest to declare.

Open Access Statement: This is an Open Access article distributed in accordance with the Creative Commons Attribution-NonCommercial-NoDerivs 4.0 International License (CC BY-NC-ND 4.0), which permits the noncommercial replication and distribution of the article with the strict proviso that no changes or edits are made and the original work is properly cited (including links to both the formal publication through the relevant DOI and the license). See: https://creativecommons.org/licenses/by-nc-nd/4.0/.

\section{References}

1. Testa L, Popolo Rubbio A, Casenghi M, et al.

Transcatheter mitral valve replacement in the transcatheter aortic valve replacement era. J Am Heart Assoc 2019;8:e13352.

2. Ejiofor JI, Hirji SA, Ramirez-Del Val F, et al. Outcomes of repeat mitral valve replacement in patients with prior mitral surgery: a benchmark for transcatheter approaches. J Thorac Cardiovasc Surg 2018;156:619-27.e1.

3. Yoon SH, Whisenant BK, Bleiziffer $\mathrm{S}$, et al. Transcatheter mitral valve replacement for degenerated bioprosthetic valves and failed annuloplasty rings. J Am Coll Cardiol 2017;70:1121-31.

4. Regueiro A, Granada JF, Dagenais F, et al. Transcatheter mitral valve replacement: insights from early clinical experience and future challenges. J Am Coll Cardiol 2017;69:2175-92.

5. Webb JG, Wood DA, Ye J, et al. Transcatheter valve-invalve implantation for failed bioprosthetic heart valves. Circulation 2010;121:1848-57.

6. Webb JG, Murdoch DJ, Boone RH, et al. Percutaneous transcatheter mitral valve replacement. J Am Coll Cardiol 2019;73:1239-46.

7. Yoon SH, Bleiziffer S, Latib A, et al. Predictors of left ventricular outflow tract obstruction after transcatheter mitral valve replacement. JACC Cardiovasc Interv 2019;12:182-93.

8. Urena M, Himbert D, Brochet E, et al. Transseptal transcatheter mitral valve replacement using balloonexpandable transcatheter heart valves: a step-by-step approach. JACC Cardiovasc Interv 2017;10:1905-19.

9. Alkhouli M, Rihal CS, Holmes DR. Transseptal techniques for emerging structural heart interventions. JACC Cardiovasc Interv 2016;9:2465-80.

10. Bapat VN, Attia R, Thomas M. Effect of valve design on the stent internal diameter of a bioprosthetic valve: a concept of true internal diameter and its implications for the valve-in-valve procedure. JACC Cardiovasc Interv 2014;7:115-27.

11. Wang DD, Guerrero M, Eng MH, et al. Alcohol septal ablation to prevent left ventricular outflow tract obstruction during transcatheter mitral valve replacement: first-in-man study. JACC Cardiovasc Interv 2019;12:1268-79.

12. Mack MJ, Leon MB, Thourani VH, et al. Transcatheter aortic-valve replacement with a balloon-expandable valve in low-risk patients. N Engl J Med 2019;380:1695-705.

13. Popma JJ, Michael Deeb G, Yakubov SJ, et al. Transcatheter aortic-valve replacement with a selfexpanding valve in low-risk patients. N Engl J Med 2019;380:1706-15.

14. Whisenant B, Kapadia SR, Eleid MF, et al. One-year outcomes of mitral valve-in-valve using the SAPIEN 3 transcatheter heart valve. JAMA Cardiol 2020;5:1245-52.

15. Gada H, Kirtane AJ, Wang K, et al. Temporal trends in quality of life outcomes after transapical transcatheter aortic valve replacement: a placement of AoRTic TraNscathetER Valve (PARTNER) Trial Substudy. Circ Cardiovasc Qual Outcomes 2015;8:338-46.

16. Blackstone EH, Suri RM, Rajeswaran J, et al. Propensitymatched comparisons of clinical outcomes after transapical or transfemoral transcatheter aortic valve replacement a Placement of Aortic Transcatheter Valves (PARTNER)-I trial substudy. Circulation 2015;131:1989-2000.

17. Biancari F, Rosato S, D'Errigo P, et al. Immediate and intermediate outcome after transapical versus transfemoral transcatheter aortic valve replacement. Am J Cardiol 2016;117:245-51.

18. Dvir D. Transseptal instead of transapical valve 
implantation: making mitral great again? JACC Cardiovasc Interv 2016;9:1175-7.

19. Yoon SH, Whisenant BK, Bleiziffer S, et al. Outcomes of transcatheter mitral valve replacement for degenerated bioprostheses, failed annuloplasty rings, and mitral annular calcification. Eur Heart J 2019;40:441-51.

20. Wang DD, Eng MH, Greenbaum AB, et al. Validating a prediction modeling tool for left ventricular outflow tract (LVOT) obstruction after transcatheter mitral valve replacement (TMVR). Catheter Cardiovasc Interv

Cite this article as: Harloff MT, Chowdhury M, Hirji SA, Percy ED, Yazdchi F, Shim H, Malarczyk AA, Sobieszczyk PS, Sabe AA, Shah PB, Kaneko T. A step-by-step guide to transseptal valve-in-valve transcatheter mitral valve replacement. Ann Cardiothorac Surg 2021;10(1):113-121. doi: 10.21037/acs-2020-mv-104
2018;92:379-87.

21. Khan JM, Babaliaros VC, Greenbaum AB, et al. Anterior leaflet laceration to prevent ventricular outflow tract obstruction during transcatheter mitral valve replacement. J Am Coll Cardiol 2019;73:2521-34.

22. Babaliaros VC, Greenbaum AB, Khan JM, et al. Intentional percutaneous laceration of the anterior mitral leaflet to prevent outflow obstruction during transcatheter mitral valve replacement: first-in-human experience. JACC Cardiovasc Interv 2017;10:798-809. 